\title{
Regulation of pulmonary fibrosis by chemokine receptor CXCR3
}

\author{
Dianhua Jiang, ${ }^{1}$ Jiurong Liang, ${ }^{1}$ Jennifer Hodge, ${ }^{1}$ Bao Lu, ${ }^{2}$ Zhou Zhu, ${ }^{1}$ Shuang Yu, ${ }^{1}$ Juan Fan, ${ }^{1}$ \\ Yunfei Gao, ${ }^{3}$ Zhinan Yin, ${ }^{3}$ Robert Homer, ${ }^{1}$ Craig Gerard, ${ }^{2}$ and Paul W. Noble ${ }^{1}$
}

\begin{abstract}
1Department of Medicine, Section of Pulmonary and Critical Care, Yale University School of Medicine, New Haven, Connecticut, USA 2Perlmutter Laboratory, Children's Hospital and Harvard Medical School, Boston, Massachusetts, USA. ${ }^{3}$ Section of Rheumatology,
\end{abstract} Yale University School of Medicine, New Haven, Connecticut, USA.

\begin{abstract}
CXC chemokine receptor 3 (CXCR3) is the receptor for the IFN- $\gamma$-inducible C-X-C chemokines MIG/CXCL9,IP-10/ CXCL10, and I-TAC/CXCL11. CXCR3 is expressed on activated immune cells and proliferating endothelial cells. The role of CXCR3 in fibroproliferation has not been investigated. We examined the role of CXCR3 in pulmonary injury and repair in vivo. CXCR3-deficient mice demonstrated increased mortality with progressive interstitial fibrosis relative to WT mice. Increased fibrosis occurred without increased inflammatory cell recruitment. CXCR3 deficiency resulted in both a reduced early burst of IFN- $\gamma$ production and decreased expression of CXCL10 after lung injury. We identified a relative deficiency in lung NK cells in the unchallenged CXCR3-deficient lung and demonstrated production of IFN- $\gamma$ by WT lung NK cells in vivo following lung injury. The fibrotic phenotype in the CXCR3-deficient mice was significantly reversed following administration of exogenous IFN $-\gamma$ or restoration of endogenous IFN- $\gamma$ production by adoptive transfer of WT lymph node and spleen cells. Finally, pretreatment of WT mice with IFN- $\gamma$-neutralizing Ab's enhanced fibrosis following lung injury. These data demonstrate a nonredundant role for CXCR3 in limiting tissue fibroproliferation and suggest that this effect may be mediated, in part, by the innate production of IFN- $\gamma$ following lung injury.
\end{abstract}

\section{Introduction}

Diffuse progressive pulmonary fibrosis occurs in a variety of clinical settings and can be life threatening (1-3). Pulmonary fibrosis can occur following environmental or occupational exposure, chronic aspiration, or in the setting of systemic illness such as autoimmune disease (scleroderma, rheumatoid arthritis, and polymyositis). Diffuse progressive pulmonary fibrosis can also occur without an evident cause or associated systemic disease, and this group of disorders has been termed idiopathic interstitial pneumonias (IIPs). The most common, as well as the most severe, form of IIP is idiopathic pulmonary fibrosis (IPF) (1). IPF is a severe, progressive, and usually fatal lung disease with an average survival of 2-5 years from diagnosis. The pathology of IPF is characterized by patchy chronic interstitial inflammation, ECM deposition, accumulation of myofibroblasts, and collapse of alveoli (1). Cystic structures in the lung periphery that occur as a result of the collapse of alveoli form a pattern that has been termed "honeycombing." It has been suggested that the unremitting fibrosis is a result of multiple microinjuries that have occurred at different times over a long period (2, 3). The mechanisms that regulate the progression of fibrosis are unknown. Several features of IPF have recently been described that might provide insights into mechanisms of pathogenesis. Fibrosis appears to progress despite a paucity of interstitial

Nonstandard abbreviations used: bronchoalveolar lavage (BAL); CXC chemokine receptor 3 (CXCR3); idiopathic interstitial pneumonia (IIP); idiopathic pulmonary fibrosis (IPF).

Conflict of interest: P. Noble is a paid consultant for Intermune Pharmaceuticals, who is developing IFN- $1 \mathrm{~b}$ for therapeutic use in patients with pulmonary fibrosis. R. Homer has received honoraria from Intermune Pharmaceuticals for lecturing at CME-sponsored programs on the pathology of idiopathic pulmonary fibrosis.

Citation for this article: J. Clin. Invest. 114:291-299 (2004).

doi:10.1172/JCI200416861. inflammation, suggesting there is an abnormality in the normal mechanisms that limit fibroproliferation after tissue injury. These abnormalities have led to speculation that IPF is the result of an abnormal fibroproliferative response to a common injury or infection in a susceptible individual.

CXC chemokine receptor 3 (CXCR3) is the receptor for CXC chemokines IFN- $\gamma$-induced protein 10-kDa (IP-10, CXCL10), monokine induced by IFN- $\gamma$ (MIG, CXCL9), and IFN-inducible T cell $\alpha$ chemoattractant (I-TAC, CXCL11), whose expression is dramatically upregulated by IFN- $\gamma(4,5)$. CXCR3 and its ligands act primarily on activated $\mathrm{T}$ and NK cells and have been implicated in mediating the effects of IFN- $\gamma$, as well as T cell-dependent antitumor responses (6-8). CXCR3 and CCR5 are preferentially expressed on Th1 cells, whereas Th2 cells favor the expression of CCR3 and CCR4 (9-12). CXCR3 ligands that attract Th1 cells can concomitantly block the migration of Th2 cells in response to CCR3 ligands, thus enhancing the polarization of effector $\mathrm{T}$ cell recruitment (13).

Recent studies have correlated the expression of CXCR3 and its ligands with a variety of diseases or pathological states. Immunoreactive CXCR3 and its ligands were expressed within atherosclerotic lesions $(14,15)$, suggesting that the expression of CXCR3 and its ligands by atheroma-associated cells plays a role in the recruitment and retention of activated $\mathrm{T}$ cells within vascular wall lesions during atherogenesis. CXCR $3^{+} \mathrm{T}$ cells were increased in blood of relapsing-remitting multiple sclerosis, and CXCL10 and CXCL9 were expressed by astrocytes in multiple sclerosis lesions (16-18) and experimental autoimmune encephalitis (19-21). CXCR3 expression has been documented on infiltrating cells in rheumatoid arthritis (22-24). Hancock and colleagues found that acute cardiac allograft rejection is accompanied by progressive intragraft production of all three ligands and by infiltration of $\mathrm{CXCR}^{+}$-activated $\mathrm{T}$ cells $(25,26)$. Additionally, mice treated 
A

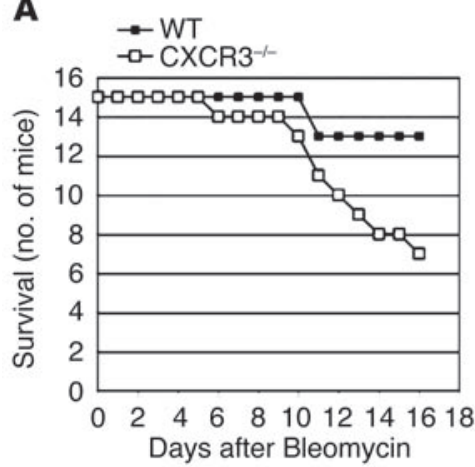

C

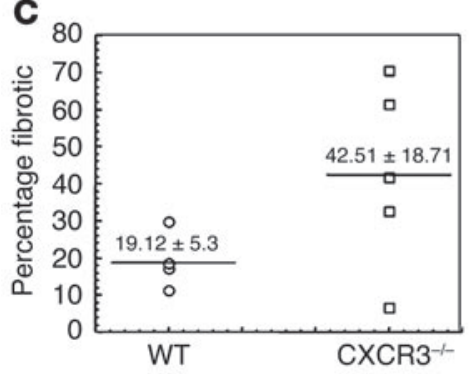

B

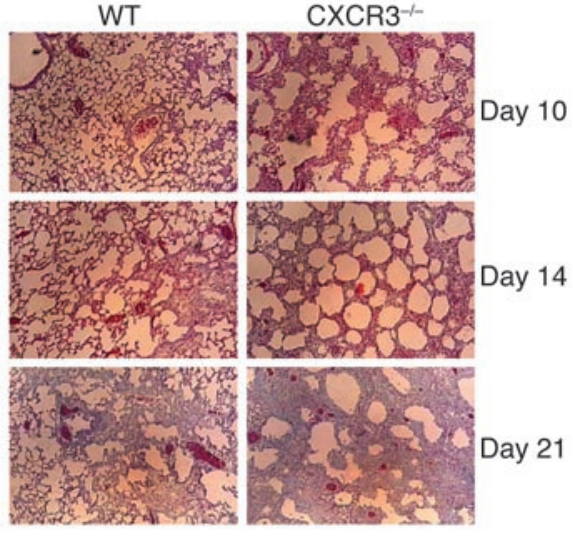

D

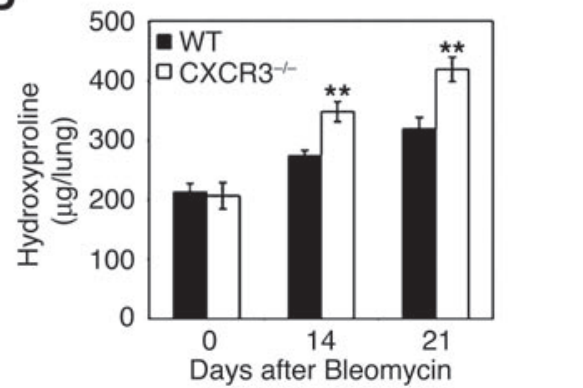

\section{Figure 1}

Increased mortality from progressive lung fibrosis in CXCR3--- mice following bleomycin-induced lung injury. (A) CXCR3--- mice and WT C57BL/6J control mice were treated intratracheally with bleomycin. By day $14,53.4 \%$ of $\mathrm{CXCR}^{-/-}$mice were dead, whereas only $13.4 \%$ mortality was seen in WT mice. (B) Lung sections of $\mathrm{CXCR}^{-1-}$ or WT mice 10, 14, and 21 days after bleomycin instillation were stained with trichrome and counterstained with hematoxylin. Magnification, $\times 100$. Seven mice in each group were examined, and similar results were observed. Note that WT mice showed typical bleomycin-induced lung injury, whereas $\mathrm{CXCR}^{-1-}$ mice exhibited dramatic collagen staining. (C) Lung fibrosis after trichrome staining was quantitated by a pathologist in a blinded manner. The fibrotic area is presented as a percentage. ${ }^{*} P=0.05$. (D) Lung tissue from $\mathrm{CXCR}^{--}$mice and WT controls 14 and 21 days after bleomycin treatment was collected and assayed for collagen content using a conventional hydroxyproline assay. This is representative of three similar experiments. ${ }^{* \star} P<0.05$. with an anti-CXCR3 mAb showed prolongation of allograft survival, and CXCR3-deficient mice showed a profound resistance to acute allograft rejection (25). Collectively, these studies have suggested a critical role for CXCR3 in recruiting activated T cells to sites of immune-mediated inflammation.

The role of CXCR3 in tissue injury and repair has not been investigated. Polarized T cell responses have been suggested to play important roles in the development of tissue fibrosis (27). Th1 cells producing IFN- $\gamma$ and IL-12 have been demonstrated to limit the development of tissue fibrosis, whereas Th2 cells producing IL-4 and IL-13 have been shown to exaggerate tissue fibrosis (27-29). CXCL10 has also been shown to ameliorate lung fibrosis following bleomycin-induced injury (30). A recent study in patients with IPF suggested that progressive lung fibrosis is associated with a relative deficiency in IFN- $\gamma$ mRNA expression (31). In this study we directly examined the role of CXCR3 in pulmonary injury, inflammation, and fibrosis in vivo. Using the well-characterized intratracheal bleomycin model of lung injury, inflammation, and fibrosis in CXCR3-deficient mice, we found that CXCR3 plays a major, nonredundant role in limiting lung fibrosis in response to noninfectious tissue injury by promoting endogenous production of IFN- $\gamma$. In addition, we identified a previously unrecognized role for CXCR3 in maintaining NK cell homeostasis in the normal, unchallenged lung.

\section{Results}

Increased mortality from progressive fibrosis in CXCR3-deficient mice following noninfectious lung injury. To examine the role of CXCR3 in noninfectious lung injury and repair bleomycin was instilled intratracheally into CXCR3-deficient mice backcrossed six generations onto the C57BL/6J background and WT littermate controls. Bleomycin initially causes an oxidant-mediated injury characterized by an inflammatory response that peaks by day 7-9.
Resolution and resultant patchy fibrosis complete the response (32). We observed that CXCR3-deficient mice were more susceptible to bleomycin-induced lung injury and suffered a significant increase in mortality relative to the WT mice (53.4\% CXCR3-deficient versus $13.4 \%$ death; Figure 1A). Histologic evaluation of the lung tissue from mice 10,14 , and 21 days after bleomycin treatment revealed that CXCR3-deficient mice developed a distinct pattern of interstitial fibrosis relative to the WT mice (Figure 1B). The WT mice showed the limited patchy fibrosis typical of bleomycin-induced lung injury, whereas the fibrosis pattern in the CXCR3-deficient mice was more severe and extensive and suggestive of the cystic honeycomb pattern observed in patients with IPF. Quantitation of fibrotic lung sections by a blinded pathologist illustrated an increase in fibrosis in the CXCR3-deficient mice compared with WT mice (Figure 1C). Furthermore, quantitation of collagen content supported the lung histology results and documented that at 21 days there were significant increases in collagen deposition in the CXCR3-deficient animals relative to $\mathrm{WT}$ controls (Figure 1D). Importantly, the fibrosis was progressive in the CXCR3-deficient animals but not the WT C57BL/6J control group (data not shown).

CXCR3-deficient mice exhibit defective lymphocyte and NK cell recruitment after noninfectious lung injury. Examination of the inflammatory response after bleomycin-induced lung injury revealed an insignificant reduction in total inflammatory cells in the bronchoalveolar lavage (BAL) fluid from CXCR3-deficient animals over 14 days as compared with WT animals (Figure 2A). Thus, the CXCR3-deficient mice developed severe progressive lung fibrosis without a significant increase in inflammatory cell recruitment. This observation led us to further examine the recruitment of specific subsets of inflammatory cells following lung injury. Differential counts of the BAL cells demonstrated fewer total lymphocytes in CXCR3-deficient mice after 


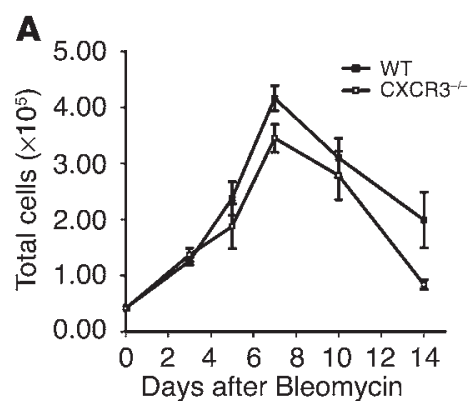

D

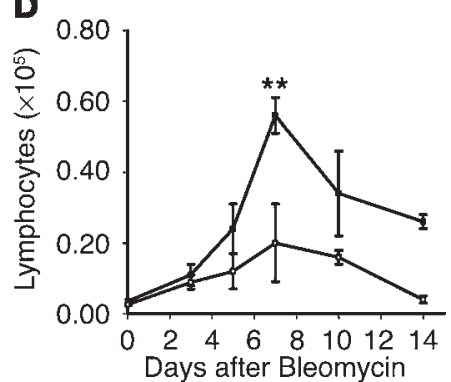

B

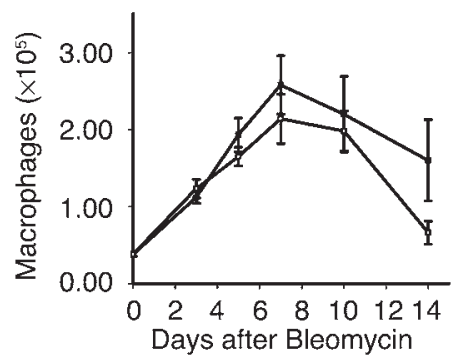

E
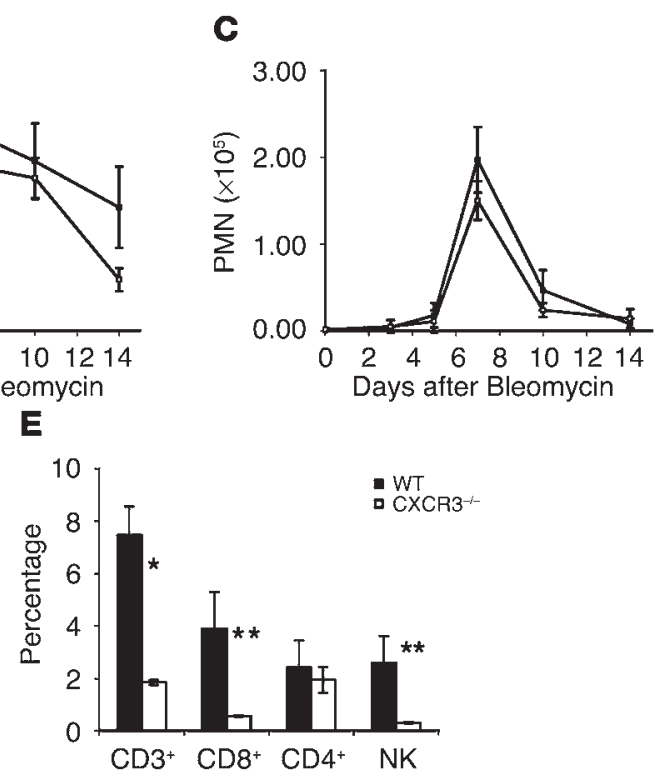

Figure 2

$\mathrm{CXCR}^{-/-}$mice exhibit a defect in lymphocyte and NK cell recruitment after lung injury. (A-D) After bleomycin treatment, BAL cells were collected on the indicated days and total cell $(\mathbf{A})$ and differential cell counting $(\mathbf{B}-\mathbf{D})$ were performed $\left(\right.$ cells $\left.\times 10^{5}\right)$. Five mice were in each group at each time point. PMN, polymorphonuclear leukocytes. ${ }^{*} P<0.05$. (E) FACS analysis of BAL cells using specific Ab's to CD3, CD8, CD4, and the NK cell marker, NK1.1. Percentages of $\mathrm{CD}^{+}, \mathrm{CD}^{+}, \mathrm{CD} 4^{+}$, and NK1.1+ cells were plotted. Five animals in each group were tested. ${ }^{*} P<0.01,{ }^{* *} P<0.05$.

bleomycin-induced lung injury at day 7 compared with WT controls (Figure 2D). The differences in macrophage and neutrophil recruitment between CXCR3-deficient and WT animals were modest (Figure 2, B and C). We next analyzed specific leukocyte populations by flow cytometry. There were significantly fewer $\mathrm{CD}^{+}$cells in the BAL fluid from the CXCR3-deficient mice after
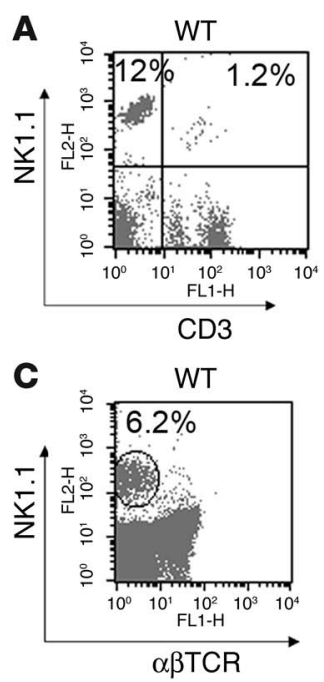
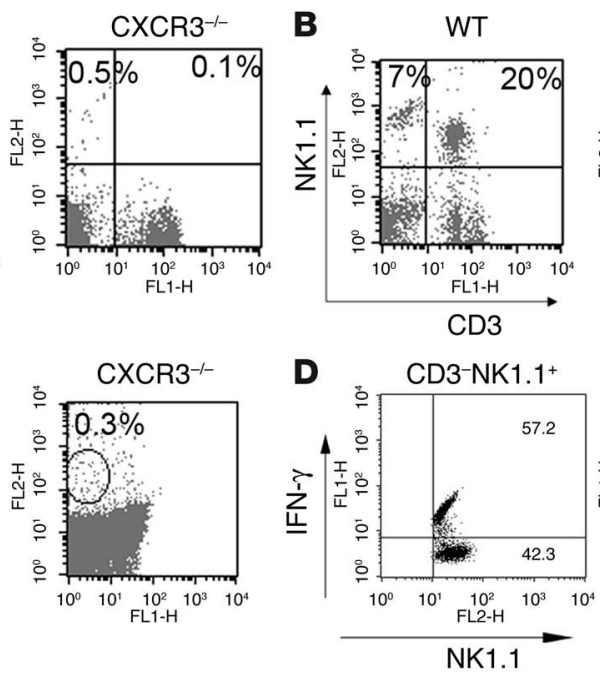
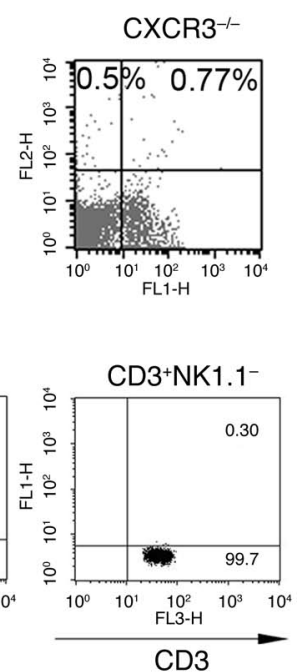

Figure 3

NK and NK T cell deficiency in CXCR3-- mice. FACS analysis of NK cells from lung, liver, and peripheral blood using specific Ab to the NK cell marker, NK1.1, and T cell markers CD3 or $\alpha \beta T C R$. (A) Single cell homogenates from unchallenged lungs of WT C57BL/6 and CXCR3 ${ }^{-1-}$ mice were stained with NK1.1 and anti-CD3e and were subject to flow-cytometric analysis. Analysis was performed on lymphocyte-gated events. The percentages of $\mathrm{NK} 1.1^{+} \mathrm{CD} 3-$ or NK1.1+CD3 ${ }^{+}$populations are indicated. Four animals in each group were tested. Similar results were obtained in four separate experiments. (B) FACS analysis of NK and NKT cells from liver. The percentage of NK1.1+CD3- or NK1.1+CD3 $3^{+}$populations are indicated. Four animals in each group were tested. Similar results were obtained in three separate experiments. (C) FACS analysis of NK cells from peripheral blood. The percentages of $\mathrm{NK}_{1.1}{ }^{+} \alpha \beta \mathrm{TCR}^{-}$population are indicated. (D) Intracellular staining for IFN- $\gamma$. Single-cell homogenates from C57BL/6 mice challenged with bleomycin for 24 hours were stained for cell surface markers NK1.1 and CD3 and for intracellular IFN- $\gamma$. After gating on $\mathrm{CD}^{+}$and $\mathrm{NK} 1.1^{+}$, the percentage of IFN- $\gamma$-producing cells from CD3-NK1.1+ and CD3+NK1.1- populations is shown. 
A

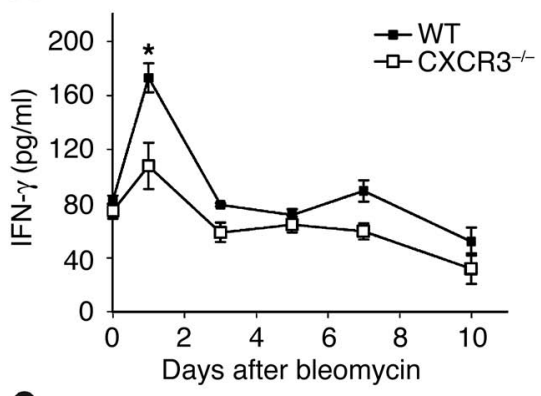

C

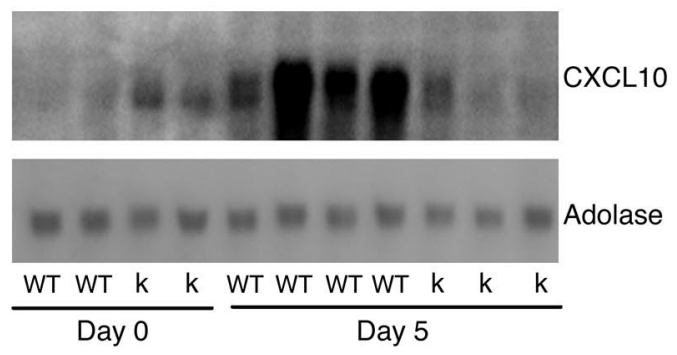

B

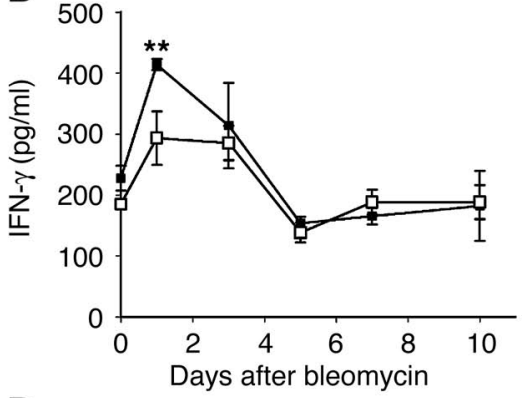

D

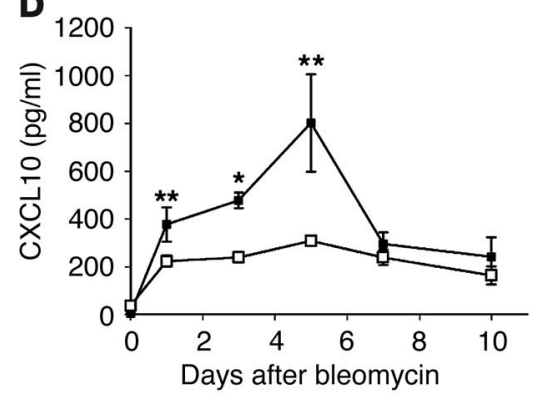

Figure 4

Reduced expression of IFN- $\gamma$ and CXCR3 ligands in CXCR3-deficient mice following bleomycin-induced lung injury. Levels of IFN- $\gamma$ protein in BAL (A) and in lung homogenates (B) in CXCR3-deficient and WT mice following bleomycin-induced lung injury were measured using ELISA. ${ }^{*} P<0.01$; ${ }^{* \star} P<0.05$. (C) Levels of CXCR3 ligand CXCL10 mRNA in the mouse lungs treated with bleomycin intratracheally at indicated days were assayed by Northern blot. Aldolase was used as an internal control for equal loading of samples. $\mathrm{k}, \mathrm{CXCR}^{-/-}$. (D) Levels of CXCR3 ligand CXCL10 protein in the mouse lungs treated with bleomycin intratracheally at indicated days were assayed with ELISA. Similar results were obtained in three separate experiments. ${ }^{*} P<0.01 ;{ }^{* \star} P<0.05$. bleomycin acute lung injury (Figure 2E). In addition, we found a selective reduction in $\mathrm{CD}^{+} \mathrm{T}$ cell recruitment to the lung in CXCR3-deficient mice (Figure 2E). We also identified a striking defect in NK cells at day 7 following bleomycin treatment. These data suggest that CXCR3 is required to recruit $\mathrm{CD}^{+} \mathrm{T}$ cells and NK cells after lung injury. We then examined leukocyte content in whole lung tissue in the unchallenged WT and CXCR3-deficient mice. Much to our surprise, we found a profound and previously unrecognized deficiency in lung NK cells in the CXCR3deficient mice. As shown in Figure 3A (see also Supplemental Figure S1A; supplemental material available at http://www.jci. org/cgi/content/full/114/2/291/DC1), CXCR3-deficient mice had markedly reduced NK cells in the unchallenged lung relative to control mice. We also examined the NK cell population by flow cytometry in liver and peripheral blood. As shown in Figure 3, there was a similar deficiency in liver (Figure 3B and Supplemental Figure 1B) and peripheral blood (Figure 3C and Supplemental Figure S1E) in the CXCR3-deficient mice as found in the lung. Dual staining for NK1.1 $1^{+}$and CD3 revealed a marked deficiency in NK-T cells in the liver (Figure 3B and Supplemental Figure S1D) and potentially in lung (Figure 3A and Supplemental Figure S1B). These data suggest that CXCR3 is required for normal homeostasis of NK and NK T cells in the unchallenged host. Importantly, we were able to demonstrate that 24 hours after lung injury, at the time when IFN- $\gamma$ production peaks (see below), CD3-NK1.1+ cells but not CD3 ${ }^{+} \mathrm{NK} 1.1^{-}$cells from lung tissue of WT C57BL/6J mice produce IFN- $\gamma$ in vivo (Figure 3D).

Reduced expression of IFN- $\gamma$ and CXCR3 ligands in CXCR3-deficient mice following lung injury. We next examined the expression of IFN- $\gamma$ and CXC chemokines following bleomycin-induced lung injury. IFN- $\gamma$ levels were increased in both BAL and lung tissue homogenates in WT mice at day 1 after bleomycin treatment (Figure 4, A and B). This transient burst in IFN- $\gamma$ production dissipated by day 3 . We found a significant reduction in the burst of IFN- $\gamma$ production following tissue injury in CXCR3-deficient mice (Figure 4, A and B). These data show, we believe for the first time, that CXCR3 is required for optimal IFN- $\gamma$ production following acute lung injury. As shown in Figure 3D, the cellular source of the innate burst of IFN- $\gamma$ is resident NK cells. We also examined CXCR3 ligand expression and found increased CXCL10 mRNA expression and protein production in lung tissue obtained from WT mice that peaked at day 5 following bleomycin lung, but not in CXCR3-deficient mice (Figure 4, C and D). These data demonstrate that CXCR3 is required for optimal IFN- $\gamma$ expression, and CXCR3-deficient mice are defective in their ability to express CXCR3 ligands following lung injury.

Exogenous IFN- $\gamma$ partially reverses the fibrotic phenotype in CXCR3deficient mice. To test the hypothesis that the transient burst of IFN- $\gamma$ after noninfectious lung injury has an important role in regulating the fibroproliferative response, we administered exogenous IFN- $\gamma$ by intramuscular injection to CXCR3-deficient mice subjected to bleomycin-induced lung injury. IFN- $\gamma$ protein can be detected in both BAL and lung tissue 24 hours after injection, and we titrated the dose delivered intramuscularly that would deliver concentrations in the BAL similar to those detected in WT mice after bleomycin treatment (data not shown). The transient administration of exogenous IFN- $\gamma$ delivered only during the first 48 hours after lung injury significantly inhibited the development of lung fibrosis as measured by quantitative assessment of lung collagen content at 21 days (Figure 5A). These data support the hypothesis that IFN- $\gamma$ limits the extent of fibrosis after injury. The incomplete inhibition in the development of tissue fibrosis by exogenous IFN- $\gamma$ suggests that CXC chemokines may have direct protective effects against tissue fibrosis independent of IFN- $\gamma$.

IFN- $\gamma$-neutralizing Ab treatment increases tissue fibrosis following noninfectious lung injury. To directly explore the role of endogenous IFN- $\gamma$ in bleomycin-induced lung injury, we administered IFN- $\gamma$-neutralizing Ab 24 hours prior to bleomycin in WT C57BL/6J mice. As shown in Figure 5B, collagen content measured at day 21 after lung injury was significantly increased in the mice treated with IFN- $\gamma$-neutralizing $\mathrm{Ab}$, but not the isotype- 
A

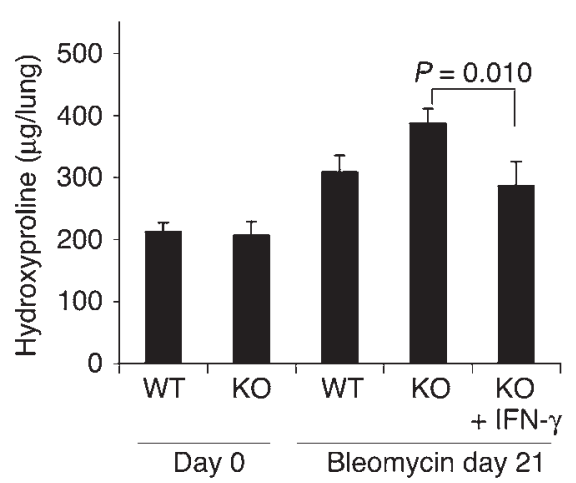

B

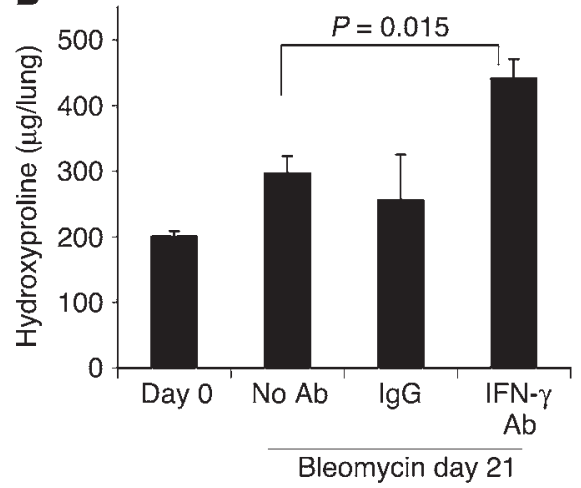

Figure 5

IFN- $\gamma$ regulates lung fibrosis. (A) Lung injury was induced in CXCR3-deficient (KO) or WT mice by intratracheal instillation of bleomycin. For one group of CXCR3-deficient mice, recombinant IFN- $\gamma$ was injected intramuscularly 20 hours before and repeated 24 and 48 hours after bleomycin treatment. On day 21 after bleomycin instillation, the lungs were harvested and assayed for collagen content. (B) Neutralizing IFN- $\gamma$ Ab worsens lung fibrosis. IFN- $\gamma$ or isotype-matched control Ig was given to $\mathrm{C} 57 \mathrm{BL} / 6 \mathrm{~J}$ mice 24 hours before bleomycin lung injury. Collagen contents were assayed on day 21 following injury in C57BL/6J mice. Six to seven mice per group were used.

matched control Ab. These data further support the concept that the endogenous production of IFN- $\gamma$ following noninfectious lung injury regulates the fibroproliferative response.

Adoptive transfer of $\mathrm{CXCR}^{+} \mathrm{LNs}$ and spleen cells restores endogenous IFN- $\gamma$ production and partially reverses the fibrotic phenotype in CXCR3-deficient mice. In an attempt to provide additional evidence for a direct role of CXCR3 and IFN- $\gamma$ in regulating the fibroproliferative response to noninfectious lung injury, we performed adoptive transfer experiments. Single cell suspensions from spleen and LNs from CXCR3-deficient and WT mice were transferred to CXCR3-deficient mice. Bleomycin was administered, and $\mathrm{CXCR}^{+}$cell recruitment, IFN- $\gamma$ production, and hydroxyproline content were evaluated. As shown in Figure 6A, we partially reconstituted $\mathrm{CXCR}^{+}$lung cells in CXCR3-deficient mice by transfer of WT cells. Additionally, adoptive transfer of $\mathrm{CXCR}^{+}$cells significantly restored IFN- $\gamma$ production in CXCR3-deficient mice (Figure 6B). Finally, the fibrotic phenotype in CXCR3-deficient mice was significantly reversed by the transfer of WT cells (Figure 6C). These data show that CXCR3 ${ }^{+}$ cells are required for the early production of IFN- $\gamma$ following noninfectious lung injury.
In summary, we have provided several lines of evidence to demonstrate a nonredundant role for CXCR3 in limiting the extent of lung fibrosis following noninfectious injury. In addition, these data suggest that endogenous IFN- $\gamma$ production occurring early following noninfectious lung injury regulates the subsequent fibroproliferative response.

\section{Discussion}

The mechanisms that regulate progressive tissue fibrosis are incompletely characterized. An important and underappreciated aspect of progressive fibrosis is a failure of endogenous inhibitory mechanisms to limit response to an acute insult (2). Here we present evidence in support of a key role for CXCR3 in limiting fibrosis following noninfectious lung injury. We found that in the absence of CXCR3, a severe, progressive, interstitial fibrosis develops in response to bleomycin-induced lung injury. The lung pathology in the CXCR3-deficient mice is distinct from that observed in WT mice following bleomycin treatment and is suggestive of the cystic honeycomb pattern observed in IPF. The progressive interstitial fibrosis occurred without an increase in lung inflammation. This dissociation between inflammation and fibrosis has recently been suggested to be characteristic of $\operatorname{IPF}(2,33)$. The phenotype observed in the CXCR3-deficient mice following lung injury suggests that CXCR3 limits the extent of fibrosis after lung injury. To further characterize this fibrotic phenotype, we examined leukocyte recruitment following lung injury and found no difference between the CXCR3-deficient mice and WT in the influx of neutrophils or monocytes. Evaluation of T cell and NK cell recruitment revealed, however, a selective decrease in $\mathrm{CD}^{+} \mathrm{T}$ cells and NK cell recruitment to the lung in CXCR3deficient mice at day 7 following lung injury. Previous studies involving CXCR3 have suggested that CXCR3-expressing cells may potentiate Th 1 responses. We therefore examined the time course of IFN- $\gamma$ production after lung injury. We observed results similar to those that have previously been reported following bleomycin lung injury; IFN- $\gamma$ production in both BAL fluid and
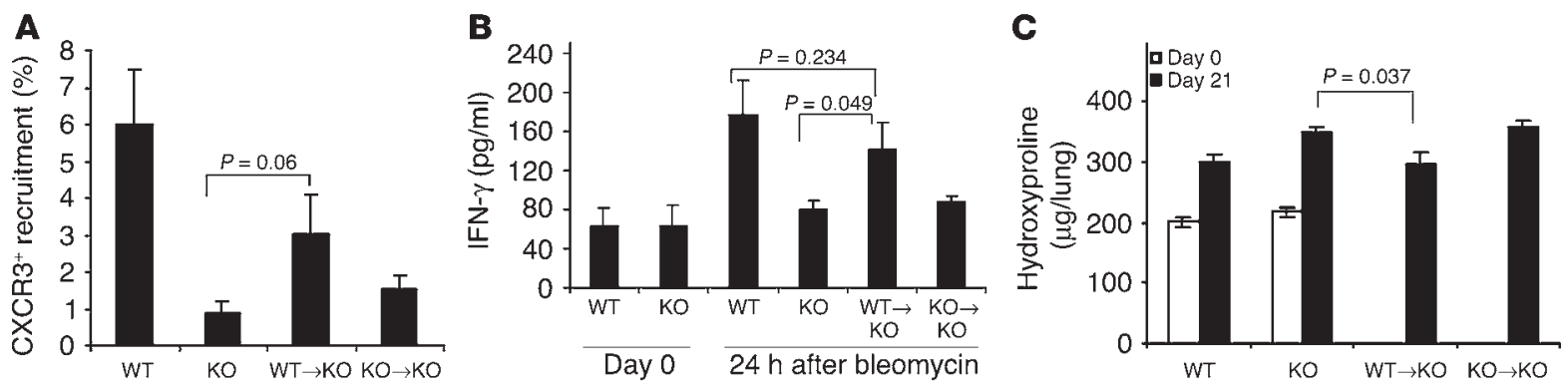

Figure 6

Adoptive transfer of $\mathrm{CXCR} 3^{+}$leukocytes partially reverses the fibrotic response. CXCR3-/- mice were injected intravenously with $\mathrm{CXCR} 3^{+}$or $\mathrm{CXCR}^{-1-}$ leukocytes isolated from spleen and LNs. Following bleomycin-induced lung injury, $\mathrm{CXCR} 3^{+}$recruitment to the lung on day 7 (A), IFN- $\gamma$ production at day 1 (B), and hydroxyproline content on day $21(\mathbf{C})$ were analyzed. $P$ values are indicated. 
lung tissue increases several-fold and peaks at 24 hours following lung injury (34). We found, however, that CXCR3-deficient mice produced significantly less IFN- $\gamma$ relative to WT mice at 24 hours after bleomycin-induced lung injury. The major finding in this study is that the early and transient burst of IFN- $\gamma$ that dissipates by day 3 has a critical role in regulating the ensuing fibroproliferative response that is measured at 21 days. We provide several lines of independent evidence in support of this hypothesis. First, the transient delivery of exogenous IFN- $\gamma$ significantly reversed the fibrotic phenotype. This is direct evident that the early burst of IFN- $\gamma$ orchestrates a program of events that influence the subsequent fibroproliferative response. This result does not prove that CXCR3 is required for the endogenous IFN- $\gamma$ production, however. Therefore, in order to provide direct evidence for a critical role for CXCR3-expressing cells in regulating the fibroproliferative response, we performed adoptive transfer experiments. We found that restoring $\mathrm{CXCR}^{+}$cells from LNs and spleens of WT mice significantly reversed the phenotype in CXCR3-deficient mice. We were able to demonstrate the presence of $\mathrm{CXCR}^{+}$cells in the BAL fluid after bleomycin treatment in the CXCR3-deficient mice that received WT immune cells. Importantly, the endogenous IFN- $\gamma$ production was restored in the CXCR3-deficient mice receiving CXCR $3^{+}$immune cells. This is strong evidence that $\mathrm{CXCR}^{+}$cells are required for generating IFN- $\gamma$ after acute lung injury. In addition, restoring CXCR $3^{+}$ cells and endogenous IFN- $\gamma$ production by adoptive transfer significantly reversed the fibrotic phenotype at 21 days. These data support our hypothesis that the defect in IFN- $\gamma$ production in the CXCR3-deficient mice contributes to the fibrotic phenotype. Finally, in order to determine if endogenous IFN- $\gamma$ production has a role in regulating the fibroproliferative response in WT mice, we treated WT mice with IFN- $\gamma$-neutralizing Ab. The IFN- $\gamma-$ neutralizing $\mathrm{Ab}$ used in this study has been shown to be effective in vivo within 6 hours of intraperitoneal administration and for a duration up to 14 days $(35,36)$. We found that treatment with IFN- $\gamma$-neutralizing Ab resulted in a significant increase in lung fibrosis at 21 days in C57BL/6J mice after bleomycin treatment relative to isotype control Ab's. Collectively, these data strongly support that the fibrotic phenotype observed in the CXCR3-deficient mice following lung injury is due to a failure to generate sufficient IFN- $\gamma$ within 24 hours to orchestrate a series of events that limit fibroproliferation.

We then turned our attention to the source of the IFN- $\gamma$ at 24 hours following lung injury. These data suggest that the source of IFN- $\gamma$ must be from an innate mechanism rather than an adaptive response, since the production occurs prior to $\mathrm{T}$ cell recruitment. To identify potential sources of IFN- $\gamma$, we examined leukocyte populations in the unchallenged lung tissue. We identified a previously unrecognized role for CXCR3 in regulating immune cell homeostasis in the naive host. The unchallenged lung was found to be deficient in NK cells. In accordance with previously published reports, approximately $10 \%$ of leukocytes in the naive lung are NK cells $(37,38)$. This readily discernible population is absent in the CXCR3-deficient lung, however. To determine if this defect was restricted to the lung we examined both NK and NK T cell populations in the liver and peripheral blood. We found that both populations were reduced in the liver and NK cells in peripheral blood of naive animals. These data show, we believe for the first time, that CXCR3 is required for NK and NK T cell homeostasis in the unchallenged host.
Several studies have shown a role for CXCR3 is that of recruiting $\mathrm{T}$ cell subsets to sites of immune-mediated inflammation $(18,39-45)$. These studies have shown that CXCR3 mediates recruitment of $\mathrm{T}$ cell subsets that are capable of producing IFN- $\gamma$. In addition, these studies have shown that inhibiting CXCR3 functions abrogates immune-mediated tissue pathology, particularly when the process is antigen driven. Thus, CXCR3 has been shown to be expressed on activated $T$ cells and mediates certain $\mathrm{T}$ cell-driven processes. Our data are the first to suggest that CXCR3 has a role in mediating immune cell homeostasis of unchallenged leukocyte populations. Furthermore, our data are the first to show that loss of CXCR3 function leads to an exaggerated tissue response to injury. Our data suggest that CXCR3 is required for the optimal production of IFN- $\gamma$ at 24 hours following nonimmune-mediated acute lung injury. There are several potential sources of the IFN- $\gamma$ production. Our data suggest that NK cells may be an important source, but we cannot exclude a role for NK T cells, $\gamma \delta \mathrm{T}$ cells, or other $\mathrm{CD}^{+} \mathrm{T}$ cells. We were not able to identify a discernible population of NK T cells in the naive lung, but it is likely that there would be more that one cellular source contributing to this innate burst of IFN- $\gamma$ production. It is clear from the data, however, that this innate burst of IFN- $\gamma$ is CXCR3 dependent. An important distinction between the findings in this study and published work on CXCR3 is that several studies have shown a critical role for CXCR3 in regulating immune-mediated recruitment of T cells, NK cells, and dendritic cells $(39,41,42,46)$. All of these studies have shown that targeting CXCR3 reduces immune cell recruitment and ameliorates the immune-mediated pathobiology. These studies suggest that targeting CXCR3-ligand interactions may be an important therapeutic intervention to prevent immune-mediated disease. Our findings are completely different and suggest a novel role for CXCR3 as an inhibitor of tissue fibroproliferation in nonimmune-mediated tissue injury. These data suggest that restoring CXCR3 function may have a therapeutic role in preventing excessive fibroproliferation. Thus, CXCR3 may have very distinct functions, depending on the nature of the insult to the host.

Several studies have shown that IFN- $\gamma$ can limit lung fibrosis caused by exogenous fibrogenic agents (47-49). Exogenous IFN- $\gamma$ has recently been suggested to improve lung function in patients suffering from progressive IPF (31). The patients that showed improved lung function following exogenous administration of IFN- $\gamma$ were found to be deficient in IFN- $\gamma$ mRNA expression relative to patients without IPF (31). More recently, a much larger clinical trial failed to reproduce the findings in the Ziesche study (3). There was a survival benefit in patients who received IFN- $\gamma$ and adhered to the treatment protocol, however, suggesting that IFN- $\gamma$ may be beneficial in some patients with IPF (3). Nevertheless, the mechanisms by which IFN- $\gamma$ may benefit patients with IPF are unknown. In this study we tested the hypothesis that CXCR3, the receptor for the IFN- $\gamma$-inducible chemokines CXCL10, CXCL9, and CXCL11, is required for IFN- $\gamma$ production following lung injury and that endogenous IFN- $\gamma$ production limits fibrosis after lung injury. We demonstrated a relative deficiency in IFN- $\gamma$ production in the CXCR3deficient mice. We provided data from several approaches that suggest that the production of IFN- $\gamma$ following noninfectious lung injury regulates the fibroproliferative response. These data place CXCR3 expression proximal to IFN- $\gamma$ production after noninfectious lung injury. 
CXCL10 has been shown to inhibit the development of bleomycin-induced lung fibrosis, suggesting that CXC chemokines can mediate antifibrotic effects of IFN- $\gamma(30)$. It is also possible that CXCL10 exerts antifibrotic effects independent of IFN- $\gamma$. CXCR3deficient mice were found to have decreased expression of CXCL10, which could contribute to the fibrotic phenotype independent of IFN- $\gamma$ effects. In contrast to the inhibitory effects of exogenous IFN- $\gamma$ on the development of lung fibrosis and the exacerbation of fibrosis in the presence of IFN- $\gamma$-neutralizing Ab's observed in this study, IFN- $\gamma$-deficient mice were recently shown to develop less fibrosis after bleomycin-induced lung injury relative to WT mice (34). This decrease in fibrosis correlated with less inflammation following lung injury. CXCL10 chemokine expression was not examined in this study. Possible explanations for these findings could be a developmental effect of constitutive IFN- $\gamma$ deficiency on lymphopoiesis as well as potential compensation from other IFNs or CXC chemokines such as CXCL10. A significant difference between the CXCR3-deficient and IFN- $\gamma$-deficient mice treated with bleomycin is that the IFN- $\gamma$-deficient mice had a blunted acute inflammatory response that was not observed in the CXCR3-deficient mice. We have no other explanation, however, for the divergent data obtained from neutralizing IFN- $\gamma$ Ab treatment and IFN- $\gamma$-deficient mice following noninfectious lung injury.

The data obtained in this study support the concept that, following tissue injury, the production of endogenous IFN- $\gamma$ serves the important function of limiting the extent of tissue remodeling and fibrosis. The mechanisms by which IFN- $\gamma$ inhibits tissue fibrosis in vivo are unknown, but in vitro studies have suggested that one mechanism can be through the inhibition of TGF- $\beta 1$-signaling pathways (50). The data presented in this study provide evidence that CXCR3 is required to limit tissue fibrosis in vivo after injury. In the absence of functional CXCR3, progressive interstitial fibrosis develops in response to lung injury with a relative deficiency in endogenous IFN- $\gamma$ production. Thus, CXCR3 appears to have an important and nonredundant role in regulating repair processes following tissue injury. A paradigm shift in the pathogenesis of IPF has recently been proposed with the suggestion that progressive fibrosis occurs as a result of pathways leading to fibroblast proliferation and ECM deposition independent of the inflammatory response (33). One potential mechanism to explain this paradigm would be a functional defect in CXCR3 expression leading to insufficient IFN- $\gamma$ production after lung injury and a failure to antagonize the profibrotic signals generated during the injury and repair process.

\section{Methods}

CXCR3-deficient mice, bleomycin administration, and BAL. CXCR3deficient mice were described previously (25). The mice have been crossed onto the C57BL/6J background for more than five generations. Age- and sex-matched WT C57BL/6J mice were purchased from The Jackson Laboratory (Bar Harbor, Maine, USA). All animal experiments were approved by the Institutional Animal Care and Use Committee at Yale University. Under anesthesia, $5 \mathrm{U} / \mathrm{kg}$ Blenoxane (Bristol-Meyers Squibb Co., Princeton, New Jersey, USA) in $25 \mu \mathrm{l}$ PBS was instilled into the mouse trachea with a $25-\mathrm{G}$ needle inserted between the cartilaginous rings of the trachea. Control animals received saline alone. The tracheostomy site was sutured, and the animals were allowed to recover.

To lavage, mice were anesthetized, and the lungs and heart were surgically exposed. The trachea was cannulated and the lungs lavaged three times with $0.8-\mathrm{ml}$ aliquots of PBS. The live cells were recovered and counted using a hemocytometer. Cytospin preparations of BAL cells were stained with Protocol Hema3 (Biochemical Sciences Inc., Swedesboro, New Jersey, USA), and differential cell counts were performed.

Histology. Seven mice in each group were sacrificed following a time course after the induction of lung injury by transection of the aorta under anesthesia. The trachea was cannulated, and the lungs inflated with $1 \mathrm{ml}$ of $10 \%$ formalin. The tissues were fixed overnight, embedded in paraffin, and sectioned for staining with trichrome.

Hydroxyproline assay. Collagen contents in lungs of four to six mice in each group were measured with a conventional hydroxyproline method (32). The ability of the assay to completely hydrolyze and recover hydroxyproline from collagen was confirmed using samples containing known amounts of purified collagen.

Fibrosis quantitation. Lung fibrosis was quantitated by a blinded pathologist as follows. Sections were stained with trichrome. Slides were sampled with a random-start systematic sampling scheme using a 6-mm-grid randomly superimposed on the slide to indicate areas to evaluate. Digitized images of the slide were obtained using a $\times 2$ objective on an Olympus $\mathrm{BH}-2$ microscope (Olympus America, Melville, New York, USA) with a Sony DXC970MD camera (Sony America, New York, New York, USA) and an IxTV capture card (IxMicro, San Jose, California, USA) on an Apple Macintosh G3 computer (Apple Computer Inc., Cupertino, California, USA). Images were then opened in Photoshop Elements (Adobe Systems Inc., San Jose, California, USA). The overall area of the lung was obtained by manual outlining. The area of the lung that was fibrotic was then outlined and the area obtained. The pixels of total versus fibrotic tissue were then summed over each lung and a percentage was obtained.

ELISA. Levels of IFN- $\gamma$ (BD Biosciences - Pharmingen, San Diego, California, USA) and CXCL1O (R\&D Systems Inc., Minneapolis, Minnesota, USA) proteins in the BAL and whole lung tissue lysates were measured with commercial ELISA kits according to the manufacturers' instructions. Lungs were harvested after perfusion to remove blood and were homogenized in $62.5 \mathrm{mM}$ Tris buffer with protease inhibitors before ELISA measurement.

Analysis of $m R N A$. Levels of $\mathrm{mRNA}$ were assessed using Northern blot analysis. Total RNA was extracted using TRIzol Reagent (Invitrogen Corp., Carlsbad, California, USA) according to the manufacturer's instructions. Twenty micrograms of total RNA was electrophoresed under denaturing conditions through a $1 \%$ formaldehyde-containing agarose gel, and RNA was transferred to Nytran hybridization filters (Schleicher and Schuell Inc., Keene, New Hampshire, USA). RNA was cross-linked to the filter by UV cross-linking (Stratagene, La Jolla, California, USA), and the blots were hybridized overnight with $10^{6} \mathrm{cpm} / \mathrm{ml}$ of ${ }^{32} \mathrm{P}-$ labeled DNA synthesized by the random prime method (New England Biolabs Inc., Beverly, Massachusetts, USA). Following hybridization, blots were washed and exposed at $-70^{\circ} \mathrm{C}$ against Kodak XAR diagnostic film. Differences in RNA loading were documented by hybridizing selected blots with ${ }^{32} \mathrm{P}-$ labeled cDNA for aldolase.

Flow-cytometric analysis. Mouse lungs were harvested after perfusion to remove blood, and the lung cells were isolated by digestion in digestion buffer $(150 \mathrm{U} / \mathrm{ml}$ collagenase IV, $50 \mathrm{U} / \mathrm{ml}$ DNase I, $5 \%$ FCS in PBS) at $37^{\circ} \mathrm{C}$ for 30 minutes and minced against a filter. The lung cells or BAL cells were resuspended in PBS containing $1 \%$ BSA and $0.02 \%$ sodium azide. Nonspecific binding was blocked by incubating with $25 \mu \mathrm{g} / \mathrm{ml} \mathrm{Fc} \mathrm{Block} \mathrm{(BD}$ Biosciences - Pharmingen) for 15 minutes at $4^{\circ} \mathrm{C}$. Samples were 
incubated with Ab's to CD3, CD4, CD8, and/or NK1.1 (CD161). All Ab's were obtained from BD Biosciences - Pharmingen. Ab to CXCR3 was obtained from Zymed Laboratories Inc. (San Francisco, California, USA) and PE-conjugated secondary Ab from Jackson ImmunoResearch Laboratories Inc. (West Grove, Pennsylvania, USA). Samples were washed twice with PBS and then fixed in $2 \%$ paraformaldehyde. Flow cytometry was performed after gating on the lymphocyte population using a FACScaliber analytical flow cytometer (Becton-Dickinson Immunocytometry Systems, Mountain View, California, USA) and analyzed using CellQuest Pro software.

Intracellular staining IFN- $\gamma$. Single cell suspensions were stained for cell surface markers NK1.1 and CD3 for 30 minutes at $4{ }^{\circ} \mathrm{C}$. After fixing and permeabilizing with Cytofix/Cytoperm from BD Biosciences - Pharmingen according to manufacturer's instructions, the cells were then stained for intracellular IFN- $\gamma$ (BD Biosciences - Pharmingen). Flow cytometry was performed after gating on the lymphocyte population. Gating was then performed on $\mathrm{CD}^{+}$and $\mathrm{NK} 1.1^{+}$, and IFN- $\gamma$-producing cells from $\mathrm{CD}^{-} \mathrm{NK} 1.1^{+}$and $\mathrm{CD}^{+} \mathrm{NK} 1.1^{-}$cells were plotted.

Exogenous IFN- $\gamma$. CXCR3-deficient and control mice were treated with bleomycin as described above. IFN- $\gamma$ protein $(40,000 \mathrm{U} /$ mouse in $30 \mu \mathrm{l}$ PBS; R\&D Systems Inc.) was injected intramuscularly 20 hours before and 24 and 48 hours after bleomycin instillation. To determine the dose of IFN- $\gamma$ protein that would reconstitute comparable levels of IFN- $\gamma$ protein in the BAL of WT mice 24 hours after injury we titrated several doses and measured the IFN- $\gamma$ protein in BAL 20 hours after administration. We found that 40,000 $\mathrm{U} /$ mouse administered intramuscularly resulted in 100-200 pg/ $\mathrm{ml}$ IFN- $\gamma$ protein in BAL 20 hours after injection. Lungs were harvested at day 21 after treatment with bleomycin for analysis of collagen content as described above.
IFN- $\gamma$-neutralizing $A b$. Hamster anti-mouse IFN- $\gamma$-neutralizing Ab (36) was a generous gift from R. Schreiber of Washington University (St. Louis, Missouri, USA). The neutralizing Ab has been shown to be effective in mice for 14 days in vivo. Neutralizing Ab or control $\mathrm{Ab}(250 \mu \mathrm{g} /$ mouse $)$ was injected intraperitoneally 24 hours before bleomycin instillation. Lung injury was induced with bleomycin as described above. Collagen contents of mouse lungs 21 days after bleomycin injury were assessed with hydroxyproline assay.

Adoptive transfer. Cell suspensions of splenocytes and lymphocytes from LNs were depleted of red blood cells and injected into mice by tail-vein injection. Bleomycin-induced injury was induced 4 hours later, and the number of CXCR $3^{+}$cells recruited to the lung in response was measured 7 days after injury by flow cytometry. IFN- $\gamma$ secretion to BAL 1 day following injury was measured by ELISA, and lung collagen content was measured at day 21 by hydroxyproline assay as described above.

Statistics. Differences in measured variables between genetically altered mice and the control group were assessed using the Student's $t$ test. Data are expressed as the mean \pm SE where applicable. Statistical difference was accepted at $P$ values less than 0.05 .

\section{Acknowledgments}

This work was supported by NIH grant HL-60539 (P.W. Noble) and a Veteran's Administration Merit Review Grant (P.W. Noble).

Received for publication September 9, 2002, and accepted in revised form May 4, 2004.

Address correspondence to: Paul W. Noble, Section of Pulmonary and Critical Care Medicine, Yale University School of Medicine, TAC 441-D, New Haven, Connecticut 06520-8057, USA. Phone: (203) 785-3627; Fax: (203) 785-3826; E-mail: paul.noble@yale.edu.
1. Bjoraker, J.A., et al. 1998. Prognostic significance of histopathologic subsets in idiopathic pulmonary fibrosis. Am. J. Respir. Crit. Care Med. 157:199-203.

2. Noble, P.W. 2003. Idiopathic pulmonary fibrosis. New insights into classification and pathogenesis usher in a new era therapeutic approaches [review]. Am. J. Respir. Cell. Mol. Biol. 29(Suppl. 3):S27-S31.

3. Raghu, G., et al. 2004. A placebo-controlled trial of interferon gamma-1b in patients with idiopathic pulmonary fibrosis. N. Engl. J. Med. 350:125-133.

4. Luster, A.D., and Ravetch, J.V. 1987. Biochemical characterization of a gamma interferon-inducible cytokine (IP-10). J. Exp. Med. 166:1084-1097.

5. Arenberg, D.A., Zlotnick, A., Strom, S.R., Burdick, M.D., and Strieter, R.M. 2001. The murine CC chemokine, 6C-kine, inhibits tumor growth and angiogenesis in a human lung cancer SCID mouse model. Cancer Immunol. Immunother. 49:587-592.

6. Luster, A.D., and Leder, P. 1993. IP-10, a -C-X-Cchemokine, elicits a potent thymus-dependent antitumor response in vivo. J. Exp. Med. 178:1057-1065.

7. Cole, K.E., et al. 1998. Interferon-inducible T cell alpha chemoattractant (I-TAC): a novel non-ELR CXC chemokine with potent activity on activated $\mathrm{T}$ cells through selective high affinity binding to CXCR3. J. Exp. Med. 187:2009-2021.

8. Loetscher, M., et al. 1996. Chemokine receptor specific for IP10 and mig: structure, function, and expression in activated T-lymphocytes. J. Exp. Med. 184:963-969.

9. Strieter, R.M. 2001. Chemokines: not just leukocyte chemoattractants in the promotion of cancer. Nat. Immunol. 2:285-286.

10. Bonecchi, R., et al. 1998. Differential expression of chemokine receptors and chemotactic responsive- ness of type $1 \mathrm{~T}$ helper cells (Th1s) and Th2s. J. Exp. Med. 187:129-134.

11. Qin, S., et al. 1998. The chemokine receptors CXCR3 and CCR5 mark subsets of T cells associated with certain inflammatory reactions. J. Clin. Invest. 101:746-754.

12. Gerard, C., and Rollins, B.J. 2001. Chemokines and disease. Nat. Immunol. 2:108-115.

13. Loetscher, P., Moser, B., and Baggiolini, M. 2000. Chemokines and their receptors in lymphocyte traffic and HIV infection. Adv. Immunol. 74:127-180.

14. Mach, F., et al. 1999. Differential expression of three $\mathrm{T}$ lymphocyte-activating $\mathrm{CXC}$ chemokines by human atheroma-associated cells. J. Clin. Invest. 104:1041-1050.

15. Goldberg, S.H., et al. 2001. CXCR3 expression in human central nervous system diseases. Neuropathol. Appl. Neurobiol. 27:127-138.

16. Balashov, K.E., Rottman, J.B., Weiner, H.L., and Hancock, W.W. 1999. CCR5(+) and CXCR3(+) T cells are increased in multiple sclerosis and their ligands MIP-1alpha and IP-10 are expressed in demyelinating brain lesions. Proc. Natl. Acad. Sci. U. S. A. 96:6873-6878.

17. Sorensen, T.L., et al. 1999. Expression of specific chemokines and chemokine receptors in the central nervous system of multiple sclerosis patients. J. Clin. Invest. 103:807-815.

18. Mahad, D.J., Lawry, J., Howell, S.J., and Woodroofe, M.N. 2003. Longitudinal study of chemokine receptor expression on peripheral lymphocytes in multiple sclerosis: CXCR3 upregulation is associated with relapse. Mult. Scler. 9:189-198.

19. Fischer, F.R., et al. 2000. Modulation of experimental autoimmune encephalomyelitis: effect of altered peptide ligand on chemokine and chemokine receptor expression. J. Neuroimmunol. 110:195-208.

20. Narumi, S., et al. 2002. Neutralization of IFNinducible protein 10/CXCL10 exacerbates experimental autoimmune encephalomyelitis. Eur. J. Immunol. 32:1784-1791.

21. Campbell, J.D., Stinson, M.J., Simons, F.E., and HayGlass, K.T. 2002. Systemic chemokine and chemokine receptor responses are divergent in allergic versus non-allergic humans. Int. Immunol. 14:1255-1262.

22. Agostini, C., et al. 1998. Involvement of the IP-10 chemokine in sarcoid granulomatous reactions. J. Immunol. 161:6413-6420.

23. Wedderburn, L.R., Robinson, N., Patel, A., Varsani, H., and Woo, P. 2000. Selective recruitment of polarized T cells expressing CCR5 and CXCR3 to the inflamed joints of children with juvenile idiopathic arthritis. Arthritis Rheum. 43:765-774.

24. Patel, D.D., Zachariah, J.P., and Whichard, L.P. 2001. CXCR3 and CCR5 ligands in rheumatoid arthritis synovium. Clin. Immunol. 98:39-45.

25. Hancock, W.W., et al. 2000. Requirement of the chemokine receptor CXCR3 for acute allograft rejection. J. Exp. Med. 192:1515-1520.

26. Jiankuo, M., et al. 2003. Peptide nucleic acid antisense prolongs skin allograft survival by means of blockade of CXCR3 expression directing T cells into graft. J. Immunol. 170:1556-1565.

27. Lukacs, N.W., Hogaboam, C., Chensue, S.W., Blease, K., and Kunkel, S.L. 2001. Type 1/type 2 cytokine paradigm and the progression of pulmonary fibrosis. Chest. $\mathbf{1 2 0}$ (Suppl.):5S-8S.

28. Keane, M.P., Belperio, J.A., Burdick, M.D., and 
Strieter, R.M. 2001. IL-12 attenuates bleomycininduced pulmonary fibrosis. Am. J. Physiol. Lung Cell. Mol. Physiol. 281:L92-L97.

29. Lee, C.G., et al. 2001. Interleukin-13 induces tissue fibrosis by selectively stimulating and activating transforming growth factor beta(1). J. Exp. Med. 194:809-821.

30. Keane, M.P., et al. 1999. IFN-gamma-inducible protein-10 attenuates bleomycin-induced pulmonary fibrosis via inhibition of angiogenesis. J. Immunol. 163:5686-5692.

31. Ziesche, R., Hofbauer, E., Wittmann, K., Petkov, V., and Block, L.H. 1999. A preliminary study of longterm treatment with interferon gamma- $1 \mathrm{~b}$ and lowdose prednisolone in patients with idiopathic pulmonary fibrosis. N. Engl. J. Med. 341:1264-1269.

32. Adamson, I.Y., and Bowden, D.H. 1974. The pathogenesis of bloemycin-induced pulmonary fibrosis in mice. Am. J. Pathol. 77:185-197.

33. Selman, M., King, T.E., and Pardo, A. 2001. Idiopathic pulmonary fibrosis: prevailing and evolving hypotheses about its pathogenesis and implications for therapy. Ann. Intern. Med. 134:136-151.

34. Chen, E.S., Greenlee, B.M., Wills-Karp, M., and Moller, D.R. 2001. Attenuation of lung inflammation and fibrosis in interferon-gamma-deficient mice after intratracheal bleomycin. Am. J. Respir. Cell. Mol. Biol. 24:545-555.

35. Kaplan, D.H., et al. 1998. Demonstration of an interferon gamma-dependent tumor surveillance system in immunocompetent mice. Proc. Natl. Acad. Sci. U. S. A. 95:7556-7561.
36. Schreiber, R.D., Hicks, L.J., Celada, A., Buchmeier, N.A., and Gray, P.W. 1985. Monoclonal Ab's to murine gamma-interferon which differentially modulate macrophage activation and antiviral activity. J. Immunol.134:1609-1618.

37. Klemm, A., Tschernig, T., Krug, N., and Pabst, R. 1998. Lymphocyte subsets in distinct lung compartments show a different ability to produce interferon-gamma (IFN-gamma) during a pulmonary immune response. Clin. Exp. Immunol. 113:252-257.

38. Davis, G.S., Pfeiffer, L.M., and Hemenway, D.R. 2000. Interferon-gamma production by specific lung lymphocyte phenotypes in silicosis in mice. Am. J. Respir. Cell. Mol. Biol. 22:491-501.

39. Belperio, J.A., et al. 2002. Critical role for CXCR3 chemokine biology in the pathogenesis of bronchiolitis obliterans syndrome. J. Immunol.169:1037-1049.

40. Krug, A., et al. 2002. IFN-producing cells respond to CXCR3 ligands in the presence of CXCL12 and secrete inflammatory chemokines upon activation. J. Immunol.169:6079-6083.

41. Frigerio, S., et al. 2002. Beta cells are responsible for CXCR3-mediated T-cell infiltration in insulitis. Nat. Med. 8:1414-1420.

42. Xie, J.H., et al. 2003. Ab-mediated blockade of the CXCR3 chemokine receptor results in diminished recruitment of T helper 1 cells into sites of inflammation. J. Leukoc. Biol. 73:771-780.

43. Kabashima, H., Yoneda, M., Nagata, K., Hirofuji, T., and Maeda, K. 2002. The presence of chemokine (MCP-1, MIP-1alpha, MIP-1beta, IP-10, RANTES)- positive cells and chemokine receptor (CCR5, CXCR3)-positive cells in inflamed human gingival tissues. Cytokine. 20:70-77.

44. Ferrero, E., et al. 2003. Macrophages exposed to Mycobacterium tuberculosis release chemokines able to recruit selected leucocyte subpopulations: focus on gammadelta cells. Immunology. 108:365-374.

45. Langenkamp, A., et al. 2003. Kinetics and expression patterns of chemokine receptors in human CD4+ T lymphocytes primed by myeloid or plasmacytoid dendritic cells. Eur. J. Immunol. 33:474-482.

46. Gao, P., et al. 2003. The unique target specificity of a nonpeptide chemokine receptor antagonist: selective blockade of two Th1 chemokine receptors CCR5 and CXCR3. J. Lenkoc. Biol. 73:273-280.

47. Okada, T., Sugie, I., and Aisaka, K. 1993. Effects of gamma-interferon on collagen and histamine content in bleomycin-induced lung fibrosis in rats. Lymphokine Cytokine Res. 12:87-91.

48. Hyde, D.M., Henderson, T.S., Giri, S.N., Tyler, N.K. and Stovall, M.Y. 1988. Effect of murine gamma interferon on the cellular responses to bleomycin in mice. Exp. Lung Res. 14:687-704.

49. Gurujeyalakshmi, G., and Giri, S.N. 1995. Molecular mechanisms of antifibrotic effect of interferon gamma in bleomycin-mouse model of lung fibrosis: downregulation of TGF-beta and procollagen I and III gene expression. Exp. Lung Res. 21:791-808.

50. Ulloa, L., Doody, J., and Massague, J. 1999. Inhibition of transforming growth factor-beta/SMAD signalling by the interferon-gamma/STAT pathway. Nature. 397:710-713. 